\title{
Synthesis of chrysalis-like $\mathrm{CuO}$ nanocrystals and their catalytic activity in the thermal decomposition of ammonium perchlorate
}

\author{
JUN WANG ${ }^{\mathrm{a}, \mathrm{b}, *}$, SHANSHAN HE ${ }^{\mathrm{a}}$, ZHANSHUANG LI $^{\mathrm{a}}$, XIAOYAN JING ${ }^{\mathrm{a}}$, \\ MILIN ZHANG ${ }^{\mathrm{a}}$ and ZHAOHUA JIANG ${ }^{\mathrm{b}}$ \\ ${ }^{a}$ College of Material Science and Chemical Engineering, Harbin Engineering University, \\ Harbin 150001, P.R. China \\ ${ }^{\mathrm{b}}$ College of Chemical Engineering, Harbin Institute of Technology, Harbin 150001, P.R. China \\ e-mail: junwang@hrbeu.edu.cn
}

MS received 28 December 2008; revised 4 March 2009; accepted 11 March 2009

\begin{abstract}
Chrysalis-like morphologies of $\mathrm{CuO}$ have been synthesized in large-quantity via a simple chemical deposition method without the use of any complex instruments and reagents. $\mathrm{CuO}$ nanocrystals showed a different morphology at three different temperatures, 25,60 and $100^{\circ} \mathrm{C}$. The particle size, morphology and crystal structure of the samples were characterized by transmission electron microscopy (TEM), scanning electron microscopy (SEM), X-ray diffraction (XRD) and Raman spectra. The catalytic effect of $\mathrm{CuO}$ nanoparticles on the decomposition of ammonium perchlorate (AP) was investigated by STA 409 PC thermal

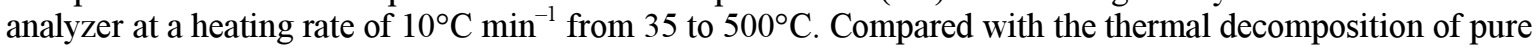
$\mathrm{AP}$, the addition of $\mathrm{CuO}$ nanoparticles decreased the decomposition temperature of $\mathrm{AP}$ by about $85^{\circ} \mathrm{C}$.
\end{abstract}

Keywords. Nanostructures; ammonium perchlorate; decomposition; catalytic activity.

\section{Introduction}

Ammonium perchlorate (AP) is one of the main oxidizing agents that have been used in various propellants. The burning behaviour of propellants is highly relevant to the thermal decomposition of AP. The catalysed thermal decomposition of ammonium perchlorate (AP) is remarkably sensitive to metal oxide additives. ${ }^{1-4}$ The underlying mechanism of nanoparticle additives in the thermal decomposition of AP still remains unclear, most likely because of the lack of a systematic study about the effects of particle size (or surface area) of $\mathrm{CuO}$ nanocrystals on the catalytic activities. For example, $\mathrm{Xu}$ et $a l^{5}$ demonstrated that $\mathrm{CuO}$ nanocrystals exhibit a particular chemical reactivity due to their high concentration of dislocations and large surface areas. In addition, the different morphologies of nanocrystals are expected to have different effects on the thermal decomposition of AP. Therefore, the synthesis and fabrication of nanostructures with different morphologies and particle size have attracted much attention for research. Well-defined $\mathrm{CuO}$ nanostructures with different morphology such as nanoparti-

\footnotetext{
*For correspondence
}

cles, ${ }^{6,7}$ nanoneedles, ${ }^{8}$ nanoshuttles, ${ }^{9}$ nanoleaves, ${ }^{10}$ nanorods, ${ }^{11,12}$ nanoribbons, ${ }^{13,14}$ nanowires, ${ }^{15-17}$ nanoplatelets ${ }^{18}$ have been synthesized successfully by a series of methods.

In this paper, $\mathrm{CuO}$ nanocrystals were synthesized by the chemical deposition method. With the different reaction temperature, $\mathrm{CuO}$ nanocrystals were prepared to show different morphologies. Moreover, with an initial reaction temperature varying from $25^{\circ} \mathrm{C}$ to $100^{\circ} \mathrm{C}, \mathrm{CuO}$ nanocrystals of different particle size and morphology were synthesized. However, with an initial reaction temperature of $60^{\circ} \mathrm{C}$, we found that the resulting products are monodispersed in large quantity and exhibit a chrysalis-like nanocrystalline monoclinic structure. The chrysalis-like $\mathrm{CuO}$ nanocrystals were studied as an additive for promoting the thermal decomposition of ammonium perchlorate (AP). With the addition of $\mathrm{CuO}$, the thermal decomposition temperature of AP decreased.

\section{Experimental}

\subsection{Synthesis of sample}

All the reagents used in this synthesis were of analytical grade and used as received without further 
purification. The typical reaction process for the synthesis of $\mathrm{CuO}$ nanocrystals include: $0.02 \mathrm{M}$ $\mathrm{Cu}\left(\mathrm{NO}_{3}\right)_{2}$ solution was prepared by dissolving $\mathrm{Cu}\left(\mathrm{NO}_{3}\right)_{2} \cdot 2 \mathrm{H}_{2} \mathrm{O}$ in $500 \mathrm{ml}$ distilled water. Then, $0.5 \mathrm{~g} \mathrm{NaOH}$ was added to the solution under constant stirring at three different temperatures, 25, 60 and $100^{\circ} \mathrm{C}$. The reaction lasted for $10 \mathrm{~min}$ forming a blue or black aqueous solution which was then heated and refluxed with continuous stirring at $100^{\circ} \mathrm{C}$ for $30 \mathrm{~min}$ in a three-necked refluxing pot. During refluxing, the temperature of the solution was controlled by manually inserting an adjustable thermocouple into the refluxing pot. The resultant precipitates were washed with distilled water several times and dried at room temperature.

\subsection{Characterization of sample}

The structure and crystal phases were characterized by power X-ray diffraction (PXRD) with $\mathrm{CuK} \alpha$ radiation, wavelength $\lambda=1.54178 \AA$ (Rigaku D/MaxIIIA). Raman measurement was performed using a Renishaw 1000 model confocal microscopy Raman spectrometer. Morphology and structure of the $\mathrm{CuO}$ nanocrystals were characterized using scanning electron microscopy (SEM, JSM-6480A) and transmission electron microscopy (TEM, PHILIPS CM $200 \mathrm{FEG}, 160 \mathrm{kV})$. The thermal behaviour of the $\mathrm{CuO}$ nanocrystals was determined using a STA 409 PC thermal analyzer at a heating rate of $10^{\circ} \mathrm{C} \mathrm{min}^{-1}$

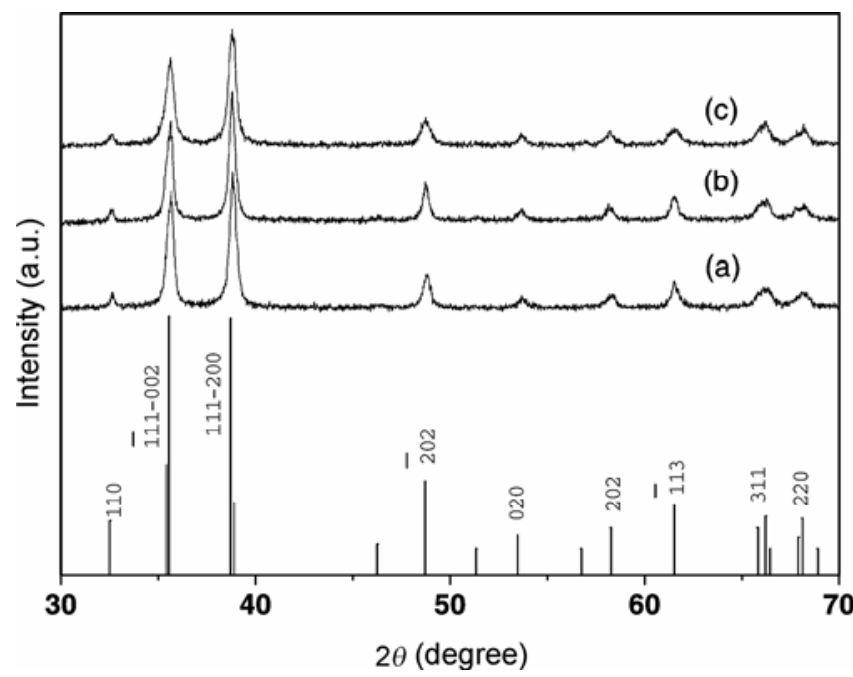

Figure 1. $\mathrm{XRD}$ pattern of the $\mathrm{CuO}$ nanostructures at different reaction temperature: (a) $25^{\circ} \mathrm{C}$; (b) $60^{\circ} \mathrm{C}$; (c) $100^{\circ} \mathrm{C}$. The $(\mid)$ vertical lines indicate the position and relative intensity of 48-1548 JCPDS card file diffraction peaks for the monoclinic phase. from 35 to $500^{\circ} \mathrm{C}$ in an argon atmosphere and under ambient atmospheric pressure.

\subsection{Catalytic activity measurements}

To evaluate the catalytic activity of the as-prepared $\mathrm{CuO}$ nanocrystals, thermogravimetric and different tial scanning calorimetric analysis techniques were applied to investigate the thermal decomposition of a mixture of $\mathrm{NH}_{4} \mathrm{ClO}_{4}$ and as-prepared $\mathrm{CuO}$ nanocrystals from 35 to $500^{\circ} \mathrm{C}$ under an argon atmosphere, in which the $\mathrm{CuO}$ powders and $\mathrm{NH}_{4} \mathrm{ClO}_{4}$ were thoroughly mixed in mass proportion of $2: 98$. The measurements were performed on a STA 409 PC thermal analyzer at a heating rate of $10^{\circ} \mathrm{C} \mathrm{min}^{-1}$.

\section{Results and discussion}

\subsection{Crystal structure and morphology of the as-synthesized $\mathrm{CuO}$}

The structure and chemical composition of all $\mathrm{CuO}$ samples synthesized in this work are confirmed with $\mathrm{XRD}$. A typical XRD pattern of all $\mathrm{CuO}$ samples is shown in figure 1 . The major peaks located at $2 \theta$ values of $30-70^{\circ}$ correspond to the characteristic diffractions of monoclinic phase $\mathrm{CuO}$ (SG: $C 2 / c$; $a_{0}=4.688 \AA, b_{0}=3.423 \AA, c_{0}=5.132 \AA, \beta=99.50^{\circ}$; JCPDS 48-1548). No other peak is observed belonging to any impurity, such as $\mathrm{Cu}(\mathrm{OH})_{2}, \mathrm{Cu}_{2} \mathrm{O}$, indicating the high purity of the obtained products.

Figure 2 shows the same magnification for SEM image of all $\mathrm{CuO}$ samples synthesized in this work. When the initial reaction temperature varied from $25^{\circ} \mathrm{C}$ to $100^{\circ} \mathrm{C}, \mathrm{CuO}$ nanocrystals with different particle sizes are synthesized. With an initial reaction temperature of $25^{\circ} \mathrm{C}$, a large number of $\mathrm{CuO}$ flower nanostructures agglomerates formed which are shown in figure $2 \mathrm{a}$. When the initial reaction temperature increased to $60^{\circ} \mathrm{C}$, well organized and homogenous chrysalis-like $\mathrm{CuO}$ nanostructures formed as shown in figure $2 \mathrm{~b}$. With a further increase of temperature, the morphology of $\mathrm{CuO}$ nanostructures changes slightly. When the initial reaction temperature reached $100^{\circ} \mathrm{C}$, the particle sizes of the $\mathrm{CuO}$ nanostructures becomes smaller and their length shorter, as shown in figure $2 \mathrm{c}$.

Figure 3 shows a different magnification for the TEM image of a sample with an initial reaction temperature of $60^{\circ} \mathrm{C}$. Figures $3 \mathrm{a}$ and $\mathrm{b}$ show the repre- 
sentative TEM images of the as-prepared $\mathrm{CuO}$ aggregates. From figure $3 \mathrm{a}$ it can be seen that the product is composed of mostly ( $95 \%)$ chrysalislike aggregates. As seen in figure $3 \mathrm{~b}$, aggregates
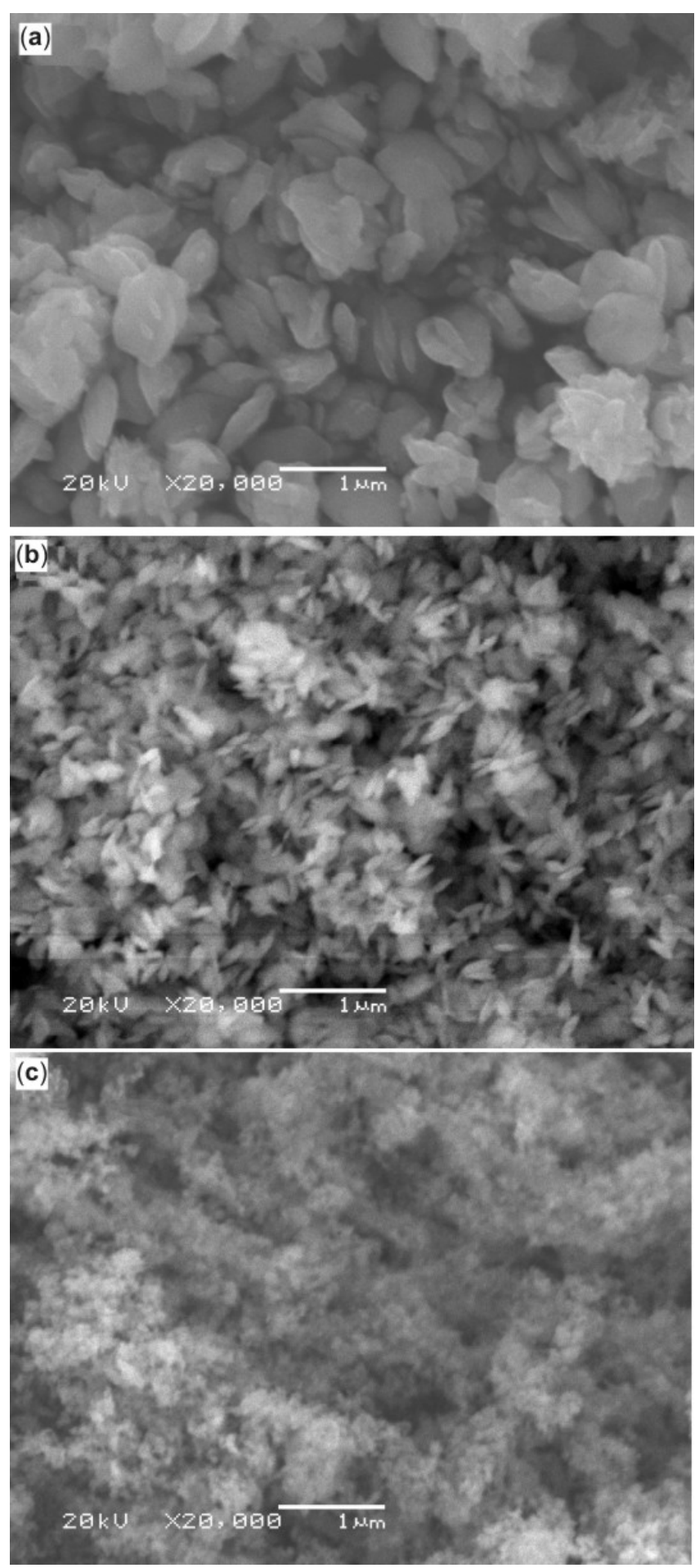

Figure 2. The same magnification SEM image of the $\mathrm{CuO}$ nanostructures at different reaction temperature: (a) $25^{\circ} \mathrm{C}$; (b) $60^{\circ} \mathrm{C}$ and (c) $100^{\circ} \mathrm{C}$. are $80-100 \mathrm{~nm}$ in breadth and $200-250 \mathrm{~nm}$ in length. With careful observation, figure $3 \mathrm{c}$ shows that the sample is composed of relatively uniform small particles. A high resolution TEM (HRSEM) image of nanoparticles, as shown in figure $3 \mathrm{~d}$, the lattice spacing of $0.25 \mathrm{~nm}$ corresponds to a $d$ spacing of [110] crystal planes. The corresponding FFT pattern (inset in figure 3d) is consistent with the HRTEM observation

Raman spectroscopy, has been widely used as a sensitive probe to investigate the microstructure, namely the local atomic arrangement and vibration, of the nano-sized materials. ${ }^{19,20}$ Figure 4 shows the Raman spectra of the as-prepared chrysalis-like $\mathrm{CuO}$ nanocrystals. The Raman spectra reveal three main phonon modes in the top of the chrysalis-like $\mathrm{CuO}$ nanocrystals, at 276, 327 and $608 \mathrm{~cm}^{-1}$. The Raman peaks are also broadened; the peak at $276 \mathrm{~cm}^{-1}$ can be assigned to the $\mathrm{Ag}$ mode, while the peaks at 327 , $608 \mathrm{~cm}^{-1}$ can be assigned to the $\mathrm{Bg}$ modes. No $\mathrm{Cu}_{2} \mathrm{O}$ modes $^{21}$ are present, demonstrating the single phase property of our chrysalis-like $\mathrm{CuO}$ nanocrystals.

\subsection{Catalytic properties of the $\mathrm{CuO}$ chrysalis-like nanostructures}

To study the catalytic properties of the chrysalis-like $\mathrm{CuO}$ nanocrystals, the thermal decomposition of the mixture of as-prepared $\mathrm{CuO}$ and $\mathrm{NH}_{4} \mathrm{ClO}_{4}$ is studied. As shown in figure $5 \mathrm{~b}$, the thermal decomposition of pure AP is divided into two exothermic phases: a lower temperature exothermic phase between 310 and $350^{\circ} \mathrm{C}$, and a higher temperature exothermic phase between $360^{\circ} \mathrm{C}$ and $400^{\circ} \mathrm{C}$. Figure 5a shows the DSC curve of the mixture of as-prepared $\mathrm{CuO}$ and $\mathrm{NH}_{4} \mathrm{ClO}_{4}$ which displays two peaks at 244.4 and $313.8^{\circ} \mathrm{C}$. It is clear that the addition of nano-sized $\mathrm{CuO}$ to AP slightly decreases the crystallographic transition temperature of AP, incorporates two small separated exothermic peaks of AP into a strong exothermic peak of AP, and drastically reduces the temperature of the second exothermic peak, indicating a strong catalytic activity on the thermal decomposition of AP

Further catalytic effect of nano-sized $\mathrm{CuO}$ on AP thermal decomposition is carried out by TG. The TG curves for pure AP and AP in the presence of $\mathrm{CuO}$ nanoparticles are shown in figure 6 . Figure $6 \mathrm{a}$ exhibits only one weight loss step and figure $6 \mathrm{~b}$ exhibits two weight loss steps, which correspond to the exothermic peaks of the DSC curves. The 

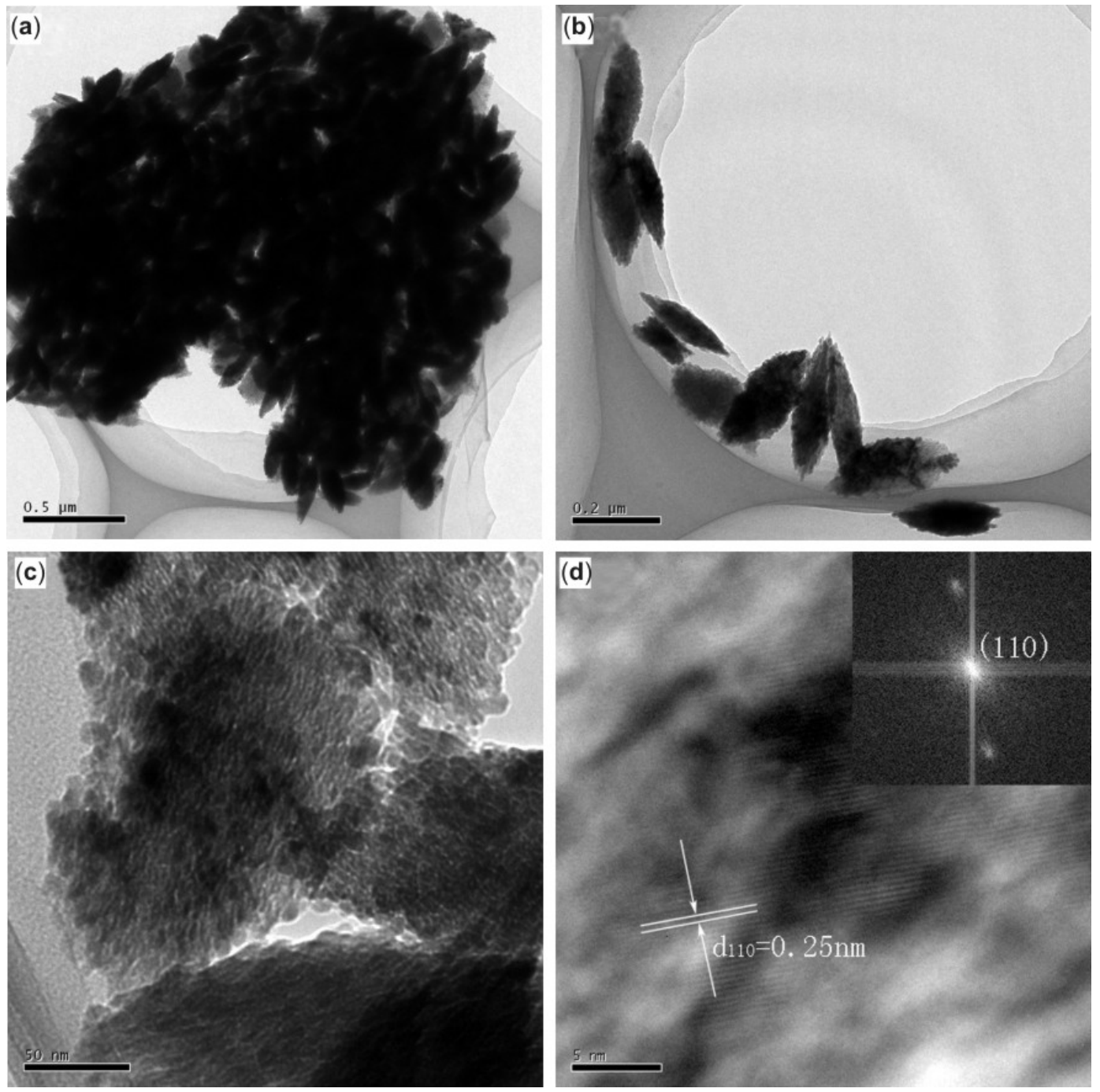

Figure 3. a-c, The different magnification TEM image of the sample with an initial reaction temperature of $60^{\circ} \mathrm{C}$; (d) HRTEM image of the sample showing the difference between two lattice fringes, which is about $0.25 \mathrm{~nm}$. Corresponding FFT pattern (inset) is consistent with the HRTEM observation.

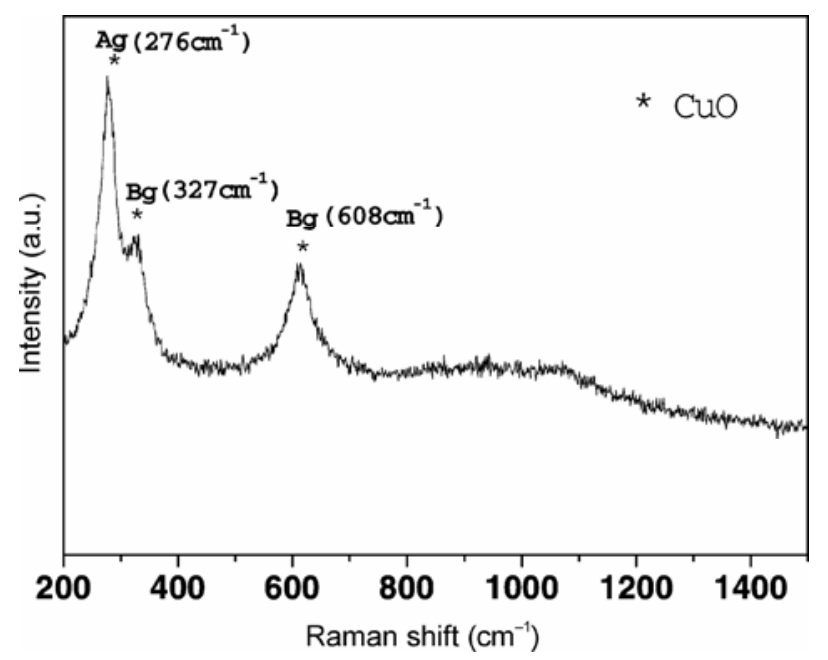

Figure 4. The Raman spectrum of the chrysalis-like $\mathrm{CuO}$ nanocrystals. onsets of thermal decomposition of the two samples are about $300^{\circ} \mathrm{C}$, while the end temperatures are about 326 and $411^{\circ} \mathrm{C}$, respectively. Adding 2 wt. \% $\mathrm{CuO}$ nanoparticles allows a decrease of the decomposition temperature of AP by $85^{\circ} \mathrm{C}$. These data indicate that addition of $\mathrm{CuO}$ nanostructures in $\mathrm{AP}$ lead to a significant reduction of the final decomposition temperature of AP.

\section{Conclusion}

In conclusion, chrysalis-like morphologies of $\mathrm{CuO}$ have been synthesized in a large-quantity via a simple solution process without the use of any complex instruments and reagents. $\mathrm{CuO}$ nanocrystals showed a different morphology at three different temperatures, 


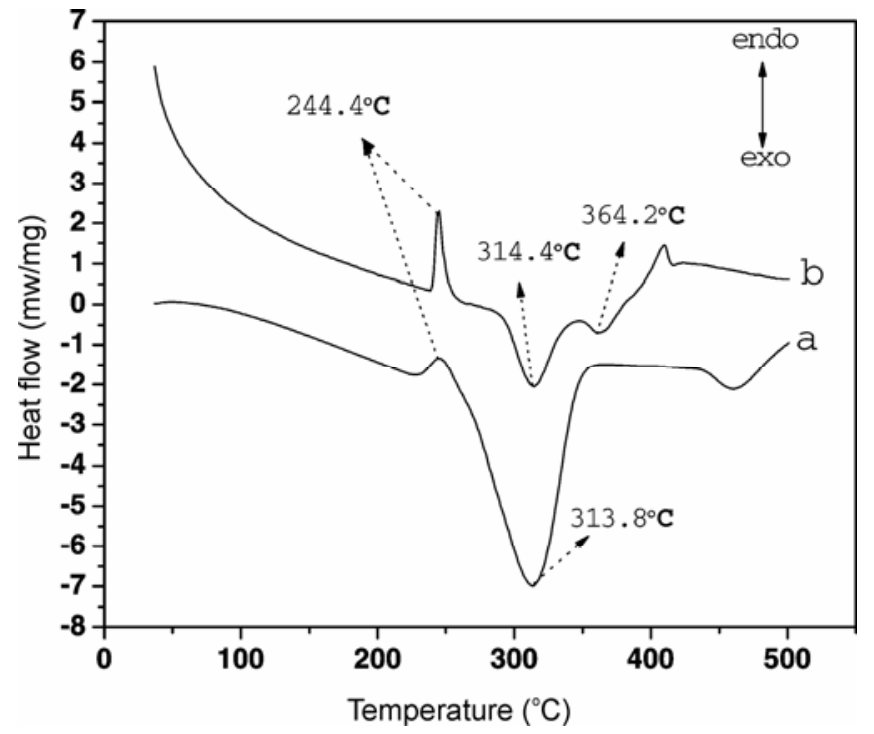

Figure 5. DSC curves for: (a) $\mathrm{AP}+$ chrysalis-like $\mathrm{CuO}$ nanocrystals; (b) pure AP.

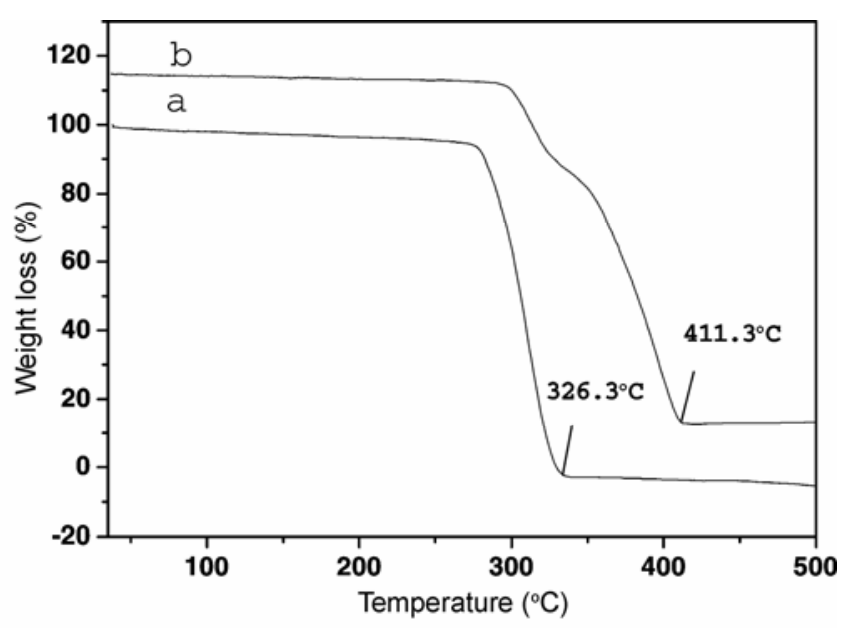

Figure 6. TG curves for: (a) $\mathrm{AP}+$ chrysalis-like $\mathrm{CuO}$ nanocrystals; (b) pure AP.

25, 60 and $100^{\circ} \mathrm{C}$. However, the optimal conditions to prepare chrysalis-like $\mathrm{CuO}$ are as follows: At an initial reaction temperature of $60^{\circ} \mathrm{C}$, we found that the resulting products are monodispersed in large quantity and exhibit a chrysalis-like nanocrystalline nature of monoclinic structure. Moreover, the addition of nano-sized $\mathrm{CuO}$ merges two exothermic peaks of AP (ammonium perchlorate) thermal decomposition into one peak, greatly reducing the high temperature decomposition peak, and drastically increasing the apparent decomposition heat of AP. Hence, there is a great potential for the use of nano-sized $\mathrm{CuO}$ in catalytic decomposition of $\mathrm{AP}$.

\section{Acknowledgements}

We gratefully acknowledge the support of this research by the Key Technology R\&D program of Heilongjiang Province (no.TB06A05), Science Fund for Young Scholar of Harbin City (no. 2004AFQXJ038) and basic research fund for Harbin Engineering University (no. mzj07076).

\section{References}

1. Survase D V, Gupta M and Asthana S N 2002 Progress in crystal growth and characterization of materials, vol 45, p. 161

2. Yanping Wang, Junwu Zhu, Xujie Yang, Lude Lu and Xin Wang 2005 Thermochim. Acta 437106

3. Guorong Duan, Xujie Yang, Jian Chen, Guohong Huang, Lude Lu and Xin Wang 2007 Powder Technol. 17227

4. Lijuan Chen, Liping Li and Guangshe Li $2008 \mathrm{~J}$. Alloys and Compounds 464532

5. Yanyan Xu, Dairong Chen, Xiuling Jiao and Keyan Xue 2007 Mater. Res. Bull. 421723

6. Premkumar T and Geckeler K E 2006 J. Phys. Chem. Solids 671451

7. Yueming Li, Jing Liang, Zhanliang Tao and Jun Chen 2008 Mater. Res. Bull. 432380

8. Yueli Liu, Lei Liao, Jinchai Li and Chunxu Pan 2007 J. Phys. Chem. C111 5050

9. Yange Zhang, Shutao Wang, Xuebing Li, Liyong Chen, Yitai Qian and Zude Zhang 2006 J. Crystal Growth 291196

10. Junwu Zhu, Huiping Bi, Yanping Wang, Xin Wan, Xujie Yang and Lude Lu 2008 Mater. Chem. Phys. 10934

11. Qi Liu, Yongye Liang, Hongjiang Liu, Jianming Hong and Zheng Xu 2006 Mater. Chem. Phys. 98519

12. Hongmei Xiao, Luping Zhu, Xianming Liu and Shaoyun Fu 2007 Solid State Commun. 141431

13. Xinyu Song, Haiyun Yu and Sixiu Sun $2005 \mathrm{~J}$. Colloid and Inter. Sci. 289588

14. Hongwei Hou, Yi Xie and Qing Li 2005 Crystal Growth Design 5201

15. Wenzhong Wang, Yan Zhuang and Lin Li 2008 Mater. Lett. 621724

16. Yikun Su, Chengmin Shen, Haitao Yang, Hulin Li and Hongjun Gao 2007 Transactions of Nonferrous Metals Soc. of China $\mathbf{1 7} 783$

17. Dinesh Pratap Singh, Animesh Kumar Ojha and Onkar Nath Srivastava 2009 J. Phys. Chem. C113 3409

18. Guifu Zou, Hui Li, Dawei Zhang, Kan Xiong, Chao Dong and Yitai Qian 2006 J. Phys. Chem. B110 1632

19. Yoshikawa M, Mori $Y$, Obata H, Maegawa M, Katagiri G, Ishida H and Ishitani A 1995 Appl. Phys. Lett. 67694

20. Yu T, Zhao X, Shen Z X, Wu Y H and Su W H 2004 J. Cryst. Growth 268590

21. Reimann K and Syassen K 1989 Phys. Rev. B Condens Matter 3911113 